\title{
Circuit
}

Musiques contemporaines

\section{Chronologie des articles, entrevues et commentaires radiophoniques}

Volume 5, numéro 1, 1994

Gilles Tremblay : réflexions

URI : https://id.erudit.org/iderudit/902097ar

DOI : https://doi.org/10.7202/902097ar

Aller au sommaire du numéro

Éditeur(s)

Les Presses de l'Université de Montréal

ISSN

1183-1693 (imprimé)

1488-9692 (numérique)

Découvrir la revue

Citer ce document

(1994). Chronologie des articles, entrevues et commentaires radiophoniques. Circuit, 5(1), 73-76. https://doi.org/10.7202/902097ar d'utilisation que vous pouvez consulter en ligne.

https://apropos.erudit.org/fr/usagers/politique-dutilisation/ 


\section{CHRONOLOGIE DES ARTICLES, ENTREVUES ET COMMENTAIRES RADIOPHONIQUES ${ }^{(1)}$}

Thèse analytique sur la Messe de Guillaume de Machaut, Conservatoire National Supérieur de Musique de Paris, 1956. (Archives personnelles de G.T.).

« Hommage à Messiaen», Melos, $n^{\circ} 25$, décembre 1958, p. 392.

« Les sons en mouvement (hommage à Varèse) 》, Liberté, sept.-oct. 1959, pp. 297. 303.

-Vers une nouvelle écoute / (texte d'une conférence présentée aux J.M.C. le 31 juillet 1961 et au Centre Social de I'U de M en mars 1966) in: Raoul Duguay, Musiques du Kebek, Éditions du Jour, Montréal, 1971, pp. 284-288.

« Ma bibliothèque idéale. Émission radiophonique, 26 août 1961. Réalisateur, Gilbert Picard, R.-C. (Texte provenant des Archives personnelles de G.T.).

« Un compositeur nous parle. "Entrevue de R. Daudelin avec Gilles Tremblay, Journal des Jeunesses Musicales, janvier 1962.

«Musique len marge du concert de musique électronique et concrète, JMC, 23 janvier 1962), Maintenant, no 3, mars 1962, p. 113.

(1) L'astérisque renvoie aux articles publiés dans ce numéro.
« Stravinsky \& Émission radiophonique, 28 août 1962, R.-C. (Texte provenant des Archives personnelles de G.T.).

- La musique classique en Amérique: II Amérique latine, 2) États-Unis, 3) Une musique naissante : la musique canadienne. Série radiophonique L'Homme américain 1963. Réalisateur, Fernand Ouellette, R.-C. (Texte provenant des Archives personnelles de G.T.I.

«Entrevue avec Gilles Tremblay », Bulletin du Collège de l'Assomption, mai 1963.

- La musique française de clavier: Messiaen Boulez. » Emission radiophonique, 15 juin 1963, R.C. (Texte provenant des Archives personnelles de G.T.).

« Propos de Serge Garant et de Gilles Tremblay sur Olivier Messiaen. Émission radiophonique. Archives sonores de R.-C. 630822. (Transcription de l'auteure).

Commentaires sur les œuvres présentées durant les 33 émissions de la série radiophonique Festival, oct. 1963 à juin 1964. (Textes provenant des Archives personnelles de G.T.).

- Le Conservatoire au Palais du Commerce! Et les foires agricoles à l'Université », Le Devoir, 8 juin 1964.

Présentation des Trois pelites liturgies de Messiaen. Émission radiophonique, février 1966, R.-C. (Texte provenant des Archives personnelles de G.T.).
"A travers le temps ef l'espace: les bruits ». Émission radiophonique. Réalisateur, Olivier Mercier-Gouin. Archives sonores de R.-C. 660723 et 660806 . (Transcription de l'auteure).

* Entrevue d'Andrée Paul avec Gilles Tremblay », Le Quartier Latin, 24 nov. 1966.

« Hommage à Monteverdi ». Série radiophonique Documents. Réalisateur, Fernand Ouellette. Archives sonores de R.C. 670526. (Transcription de l'auteure).

Présentation des œuuvres de Schœnberg, Webern, Messiaen et Garant. Série radiophonique Musique de notre siècle (première émission). Réalisateur, Claude Garneau. Archives sonores de R.-C. 670914. (Transcription de l'auteure).

Présentation de Déserts de Varèse et la musique des rites tibétains au Sikkim. Série radiophonique Musique de notre siècle , 21 déc. 1967. Réalisatrice, Aline Legrand. (Texte provenant des Archives personnelles de G.T.).

Propos sur la musique concrète. Série radiophonique Documents. Réalisateur, Fernand Ouellette. Archives sonores de R.C. 680223. (Transcription de l'auteure).

Discours lors de réception du prix Calixa Lavallée, Société St-Jean-Baptiste de Montréal, le 7 mai 1968. (Texte provenant des Archives personnelles de G.T.). 
Analyse du *Pierrot Lunaire * de Schœenberg. Série radiophonique Carnets des Arts. Réalisatrice, Aline Legrand. Archives sonores de R.-C. 681002 . (Transcription de l'auteure).

- La musique contemporaine et le public»; propos de Maurice Fleuret et Gilles Tremblay. Série radiophonique Documents. Réalisatrice, Aline Legrand. Archives sonores de R.C. 690328. (Transcription de l'auteure).

* Gilles Tremblay, un portrait * Iréponses à un court questionnairel, Musicanada 24, nov. 1969.

« Oiseau-nature, Messiaen, musique », Cahiers canadiens de musique, $n^{\circ} 1$, printempsété 1970, pp. 15-40.

* * Le bruit, prospective négative et prospective positive», in: Le bruit, $4^{\bullet}$ pollution $d u$ monde moderne, CEPQ, Cahier I, P.U.M. 1970, pp. 119.131.

* Propos de Gilles Tremblay sur un ouvrage auquel il a collaboré : Le Bruit, 4e pollution $d u$ monde moderne. Série radiophonique Horizons. Archives sonores de R.-C. 701210 . (Transcription de l'auteure).

"Serge Garant interroge Gilles Tremblay . Série radiophonique Musique de notre siècle. Réalisateur, Claude Garneau. Archives sonores de R.-C. 701019. (Transcription de l'auteure).

Critique du livre de R. Murray Schafer: The Book of Noise, Cahiers canadiens de musique, no 2, printemps-été 1971, pp. 187-188.

Propos de Gilles Tremblay sur l'art. Série radiophonique l'Art aujourd'hui. Réalisatrice, Aline Legrand. Archives sonores de R.-C. 71 1008. (Transcription de l'auteure).
«Le Sacre, avant et après *. Conférence prononcée lors du colloque sur Stravinsky à la Faculté de musique de l'Université de Toronto, $1^{\text {'r }}$ décembre 1971. (Texte provenant des Archives personnelles de G.T.).

«Du Rock au Baroque *. Entrevue avec Serge Garant et Gilles Tremblay. Émission radiophonique. Archives sonores de R.C. 720723 et 720728. (Transcription de l'auteure).

* Hélène Roy interroge Gilles Tremblay sur la musique sacrée ». Emission de télévision 5D. Archives sonores de R.C. 721210. (Transcription de l'auteure).

Discussion avec Serge Garant, Bruce Mather et Gilles Tremblay sur le Canada ef la musique d'aujourd'hui. Série radiophonique l'Art aujourd'hui. Réalisatrice, Aline Legrand. Archives sonores de R.C. 721212. (Transcription de l'auteure).

Discussion avec Serge Garant, Bruce Mather et Gilles Tremblay sur la situation du compositeur novateur. Série radiophonique l'Art aujourd'hui. Réalisatrice, Aline Legrand. Archives sonores de R.-C. 721215. (Transcription de l'auteure).

* Bali: une musique envoûtante et inépuisable ». Entrevue de Claude Gingras avec Gilles Tremblay, La Presse, 6 janvier 1973.

Propos de Gilles Tremblay sur les tendances de la musique actuelle. Série radiophonique Entretiens. Réalisateur, Fernand Ouellette. Archives sonores de R.-C. 730622. (Transcription de l'auteure).

* Sur lcare apprenti (1970) d'Henri Pousseur ». Programme SMCQ, 6 déc. 1973.

« Olivier Messiaen " in: John Vinton ed., Dictionary of Contemporary Music, N.Y. 1974, pp. 474-478.
Entrevue de Raymond BeaugrandChampagne avec Gilles Tremblay. Emission de télévision Rencontres. Archives sonores de R.-C. 740113. (Transcription de l'auteure).

Commentaires de Gilles Tremblay sur les aspects du sacré dans le monde moderne: la musique contemporaine. Émission radiophonique. Réalisateur, Fernand Ovellette. Archives sonores de R.-C. 740328 ef 740404. (Transcription de l'auteure).

* Une reconnaissance: témoignage de Gilles Tremblay sur la foi aujourd'hui ». Conférence prononcée à l'occasion des Journées universitaires de la Pensée chrétienne, 25 au 27 août 1973, U de $M$, in : Les mutations de la foi chrétienne, Fides, 1974.

* "Le point de vue d'un compositeur *, Vie spirituelle, mars-avril 1974, pp. 234-245. Article revisé sous le titre suivant: "Découvrir ce chant infiniment moderne", Communauté chrétienne, no 144, nov.-déc. 1985, pp. 573-582).

Rapport sur le séjour en Asie de 1972 présenté au Conseil des Arts, le 9 nov. 1974. (Texte provenant des Archives personnelles de G.T.).

* Entretien avec Gilles Tremblay sur I'Orient». Série radiophonique Documents. Réalisatrice, Aline Legrand. Archives sonores de R.-C. 750218 et 750225 . (Transcription de l'auteure).

* Entretien avec Gilles Tremblay sur Java ef Bali ». Série radiophonique Du monde entier au cceur du monde. Réalisateur, Yves Lapierre. Archives sonores de R.-C. 751211 . (Transcription de l'auteure).

* Les 10 ans de la SMCQ *. Programme $S M C Q, 9$ déc. 1976. 
Propos de Gilles Tremblay sur Gustav Mahler. Série radiophonique Documents. Réalisateur, Fernand Ouellette. Archives sonores de R.-C. 77011 18. (Transcription de l'auteure).

*En mémoire... (Bruno Maderna) * Programme SMCQ, 30 nov. 1978.

« Hommage da Messiaen », Variations, vol. 2, no 2, nov. 1978.

* Réflexions sur et avec Barraqué ». Programme SMCQ, 22 fév. 1979 et 11 avril 1985.

* 50 ans de musique contemporaine au Canada : propos de Gilles Tremblay ». Série radiophonique Documents. Réalisatrice, Aline Legrand. Archives sonores de R.-C. 790130. (Transcription de l'auteure).

* L'art engagé est un détournement ». Entrevue d'Yvon Paré avec Gilles Tremblay, Le Quotidien (Chicoutimi), 14 décembre 1979.

* Entrevue de Maryvonne Kendergi avec Gilles Tremblay . Coffret Radio-Canada International, ACM 12 1983. (Transcription anglaise de l'entrevue publiée dans Le Livret).

* "Même la mort se vit *, Étapes, Bulletin de la communauté chrétienne St-Albert, nov. 1980.

* Vers un choix *, Possibles, vol. 4 no 2, hiver 1980, no spécial sur « Projets du pays qui vient $\star$.

*Hommage à Maurice Martenot *. Programme SMCQ, 13 nov. 1980.

«Portrait of Gilles Tremblay *. Émission radiophonique Two New Hours, CBC Toronto. Animatrice, Norma Beecroft, 1980.
"La révolution sonore *, propos de Gilles Tremblay. Série radiophonique Actuelles. Archives sonores de R.-C. 801205801219 . (Transcription de l'auteure).

"Sur un étonnement réciproque », in: Regards sur lannis Xenakis, Stock Musique, Paris 1981, pp. 113.120.

«Art, politique et choix . Communication à une table ronde sur le développement de la culture, lors de la tournée de consultation du Ministre des Affaires culturelles, Monsieur Clément Richard, Montréal, 14 mai 1982. (Texte provenant des Archives personnelles de G.T.).

* * Le compositeur Gilles Tremblay a refusé l'Ordre du Canada * (lettre de son refus) Le Devoir, 5 juin 1982.

«L ligue canadienne de compositeurs «: commentaires de Gilles Tremblay. Émission radiophonique. Réalisateur, Richard Lavallée. Archives sonores de R.-C. 820529. (Transcription de l'auteure).

* * Hommage à Claude Vivier»: témoignage de Gilles Tremblay. Réalisateur, Gilles Poirier. Archives sonores de R.-C. 830612 . (Transcription de l'auteure).

* Claude Vivier, en mémoire, en présence», Revue de musique des universités canadiennes, no 4, 1983, pp. 3-5.

« Homélie prononcée à l'occasion de la cérémonie religieuse pour Claude Vivier $»$ le 14 avril 1983. Programme du concert commémoratif, 2 juin 1983.

* "La musique après la fin de la musique *, Entretien avec Claude lévesque et Daniel Charles, série radiophonique « Discours de la fin « de Radio-Canada. Réalisateur Fernand Ouelette. Transcription du service des droits dérivés de Radio-Canada, 1983.
"Acoustique ef forme chez Varèse *. Colloque "Varèse, 20 ans après... *, Strasbourg, oct. 1983, Revue musicale nos 383-385, déc. 1985, pp. $27-46$ et pp. $129-132$.

«L'enseignement de la composition... * point de vue des professeurs, la Scène musicale, sept.oct. 1983.

* "Composer la musique contemporaine *: conversations de Marcelle Guertin avec Gilles Tremblay ef Serge Garant, Dérives, nos 44/45, 1984, pp. 16-34.

«Un an après * Introduction au concert Vivier). Programme SMCQ, 26 avril 1984.

* "La fragilité du chant est sa force même * len réponse à la question autour du roman d'Orwell: avez-vous vécu 1984 २), Le Devoir, 17 novembre 1984.

« Un programme de musique japonaise autour de Makoto Shinohara *. Programme SMCQ, 25 fév. 1985.

* Hommage à Serge Garant * (prononcé à l'occasion des funérailles le 8 novembre 1986), Le Devoir, 18 nov. 1986. Texte intégral intitulé : «erge Garant, être de rigueur ef d'émotion », Revue de musique des universités canadiennes, no 7, 1986.

« Pour nos vingt ans *. Programme SMCQ, 4 déc. 1986.

«Du service au sévice *. Commentaire sur une critique de Claude Gingras sur une œuvre de Jean Papineau-Couture, La Presse, 24 février 1987.

* The Composers: how well do we know their music in: Beckwith, J. Cooper, D.R. ed., Hello out there I, Toronto, Institute for Canadian Music, Can Mus Document 2, 1988, pp. 173-124. 
« L'avent et la joie ». Billet pour le troisième dimanche de l'Avent, Église St-Albert-leGrand, 11 décembre 1988. (Texte provenant des Archives personnelles de G.T.).

* «Silence et liturgie *, entrevue, Liturgie, Foi et Culture, Montréal 1989.

- La voix, le Bel Canto et la création musicale • Icommunication à l'occasion du Festival international de Montréal, 11 sept. 1989). (Texte provenant des Archives personnelles de G.T.).

* Émergence de la forme dans la matière: de Varèse à Mozart. "Conférence donnée au Conservatoire de Lyon dans le cadre des Rencontres du Centre Jacques-Cartier, 5 décembre 1985. (Texte provenant des Archives personnelles de G.T.).
* Entrevue d'Anne Lauber avec Gilles Tremblay», in: M. Gagné, A. Lauber, G. Martin, A. Rioux, G. Robitaille, Sons d'aujourd'hui, Montréal, Lovise Courteau éditrice, 1990.

*Vivre avec Mozart en 1991. Communication prononcée au Conservatoire de musique du Québec. (Texte provenant des Archives personnelles de G.T.).

Conférence-dialogue avec Pierre Dansereau sur le thème de a Trace \$. Symposium de la jeune peinture au Canada, Baie Saint-Paul, 10 août 1991. (Texte provenant des Archives personnelles de G.T.).

Homélie de la Vigile de Pâques, prononcée à St-Albert-le-Grand, le 30 mars 1991. (Texte provenant des Archives personnelles de G.T.).
* Allocution prononcée lors de la remise du Prix du Québec Denise-Pelletier, le 28 octobre 1991. (Texte provenant des Archives personnelles de G.T.).

- Religion, musique et temps à travers l'expérience de la composition des Vêpres de la Vierge», Communication présentée à la Faculté de Théologie de I'Université de Montréal à l'occasion du Colloque sur Société, culture et religion dans le Montréal métropolifain, 20 mai 1992. (Texte provenant des Archives personnelles de G.T.).

La collection complète des écrits a été déposée au Centre de musique canadienne à Montréal. 